\title{
Neural crest induction in Xenopus: evidence for a two-signal model
}

\author{
Carole LaBonne* and Marianne Bronner-Fraser \\ Division of Biology, Beckman Institute 139-74, California Institute of Technology, Pasadena, CA 91125, USA \\ *Author for correspondence (e-mail clabonne@caltech.edu) \\ Accepted 7 April; published on WWW 3 June 1998
}

\section{SUMMARY}

We have investigated the molecular interactions underlying neural crest formation in Xenopus. Using chordin overexpression to antagonize endogenous BMP signaling in whole embryos and explants, we demonstrate that such inhibition alone is insufficient to account for neural crest induction in vivo. We find, however, that chordin-induced neural plate tissue can be induced to adopt neural crest fates by members of the FGF and Wnt families, growth factors that have previously been shown to posteriorize induced neural tissue. Overexpression of a dominant negative XWnt-8 inhibits the expression of neural crest markers, demonstrating the necessity for a Wnt signal during neural crest induction in vivo. The requirement for
Wnt signaling during neural crest induction is shown to be direct, whereas FGF-mediated neural crest induction may be mediated by Wnt signals. Overexpression of the zinc finger transcription factor Slug, one of the earliest markers of neural crest formation, is insufficient for neural crest induction. Slug-expressing ectoderm will generate neural crest in the presence of Wnt or FGF-like signals, however, bypassing the need for BMP inhibition in this process. A two-step model for neural crest induction is proposed.

Key words: Neural crest, Xenopus, Slug, BMP, Wnt, FGF, Chordin, Molecular interaction

\section{INTRODUCTION}

During the process of neural induction in vertebrates, the ectoderm becomes divided into several distinct tissue types including neural plate, epidermis and neural crest. Much recent work, mainly in Xenopus, has focused upon signals that can direct prospective epidermis to adopt neural fates (reviewed in Sasai and De Robertis, 1997; Weinstein and HemmatiBrivanlou, 1997). In the models of neural induction that have emerged, a relatively high level of signaling by bone morphogenetic proteins (BMPs), which are initially expressed throughout the ectoderm, is sufficient to repress neural and induce epidermal fates (Wilson and Hemmati-Brivanlou, 1995). Neural-inducing molecules such as noggin (Lamb et al., 1993), chordin (Sasai et al., 1994) and follistatin (Hemmati-Brivanlou et al., 1994), which can bind to BMPs and, presumably, prevent them from activating their receptors (Zimmerman et al., 1996; Piccolo et al., 1996; Fainsod et al., 1997), inhibit BMP signaling causing cells to adopt neural fates. This process is accompanied by a loss of BMP4 transcripts from the induced neural plate. While this model of neural induction is clearly simplistic, and many complexities are sure to emerge, it nonetheless reflects the significant progress that has been made in understanding how two of the three major ectodermally derived tissues are specified. By comparison, much less is understood about the molecular basis of neural crest induction.

The neural crest is a population of cells that is induced at the border between neural plate and epidermis. These cells eventually become migratory and populate diverse regions throughout the embryo where they give rise to most of the peripheral nervous system (PNS), epidermal pigment cells and craniofacial cartilage (Le Douarin, 1982; Hall and Hörstadius, 1988). In Xenopus, prospective neural crest cells can be identified at late gastrula stages (stage 12) by their expression of the zinc finger transcription factor Slug (Mayor et al., 1995); this expression is initially restricted to the crest-forming boundary of the neural plate, although at later stages Slug is also expressed in lateral plate mesoderm. Slug expression is also the first molecular indicator of neural crest induction in avian embryos (Nieto et al., 1994), but its expression commences later, in the closing neural folds. This may indicate a later specification for this cell type in avians.

Early experiments on neural crest induction suggested that graded amounts of the same signal could be responsible for inducing both neural and neural crest cell types. When medial archenteron roof (prospective axial mesoderm) was grafted into the blastocoel of amphibian embryos, competent ectoderm could be induced to form both neural tissue and neural crest, while lateral archenteron roof (prospective paraxial and lateral plate mesoderm) induced only neural crest (Raven and Kloos, 1945). The authors proposed that a neural/neural crest 'evocator' produced by the mesoderm would induce neural crest at low levels, while at high levels it would also induce neural tissue. Interestingly, noggin and chordin are expressed in the axial mesoderm and could represent such an 'evocator.'

More recent experiments in amphibian and avian embryos have suggested that interactions between neural plate and epidermis are sufficient to induce neural crest derivatives (Moury and Jacobson, 1990; Selleck and Bronner-Fraser, 
1995), as well as Slug expression (Dickinson et al., 1995; Mancilla and Mayor, 1996), when these tissues are juxtaposed in vitro or in vivo. In avian embryos, the ability of epidermis to induce neural crest markers in prospective neural plate can be mimicked by culturing neural plate explants in the presence of BMP4 or BMP7 (Liem et al., 1995). These experiments, together with recent results implicating graded levels of BMP signaling in neural versus epidermal cell fate decisions (Wilson and Hemmati-Brivanlou, 1995), suggest that BMPs may also specify neural crest cell fates in a concentration-dependent manner. Alternatively, the ability of epidermis to induce prospective neural plate to form neural crest may reflect a requirement for a second, non-BMP signal in this process. In addition to epidermal/neural plate interactions, there is also evidence that a signal from the mesoderm may be important for neural crest induction (Moury and Jacobson, 1990; Selleck and Bronner-Fraser, 1995; Mancilla and Mayor, 1996).

We have investigated the molecular mechanisms underlying neural crest induction, using chordin overexpression to inhibit BMP signaling in explants of prospective ectoderm from Xenopus blastulae. We find that chordin can induce low level expression of early neural crest markers in a dose-dependent manner. These levels of expression are far lower than those found endogenously, however, and such explants fail to form melanocytes, a neural crest derivative. In contrast, melanocytes and robust expression of neural crest markers, are induced when chordin-expressing explants are cultured with prospective mesoderm. This induction can be mimicked by coculturing chordin-expressing explants with those expressing eFGF (Isaacs et al., 1992) or XWnt-8 (Christian et al., 1991). Similarly, overexpression of the early neural crest marker Slug is not sufficient to induce neural crest in naive ectoderm. Slugexpressing ectoderm can give rise to neural crest, however, when cocultured with explants expressing eFGF or XWnt-8. These results suggest a model in which reduced levels of BMP signaling at the lateral edges of the neural plate provide an initial, weak specification of neural crest fate. Additional signals from the adjacent non-neural ectoderm, the underlying mesoderm or both are then required to enhance and maintain this induction. Evidence is presented that a Wnt signal is required for this process.

\section{MATERIAL AND METHODS}

\section{Preparation of embryos and explants}

Pigmented and albino embryos were obtained and fertilised in vitro by established methods, dejellied and cultured in $0.1 \times$ MMR. Staging of embryos was done according to the normal table of Nieuwkoop and Faber (1967). Embryos were injected in $1 \times$ MMR $+3 \%$ Ficoll then transferred to $0.2 \times$ MMR until further manipulation or harvesting. In vitro transcribed capped messenger RNA was dissolved in water and injected at a volume of $10 \mathrm{nl}$ per blastomere. Concentrations of injected mRNA are as noted in the text. In some whole embryo experiments, nuclear GFP (kindly provided by Robert Davis) or nuclear $\beta$-galactosidase mRNA was co-injected as a lineage tracer to assist in identifying the injected side of the embryo. Animal pole explants were harvested from late stage 9 embryos unless otherwise noted and cultured in $1 \times$ MMR containing $50 \mu \mathrm{g} / \mathrm{ml}$ gentamycin until sibling embryos reached the noted stage. Conjugates were generated by physically juxtaposing explants with watchmaker forceps and allowing them to heal on agar-coated dishes.

\section{Constructs}

Xenopus chordin in the vector pCS2+ (Sasai et al., 1994) was a kind gift of Eddy DeRobertis. pSP64T XWnt-8 (Christian et al., 1991) and dominant negative XWnt-8 in pCS2 (Hoppler et al., 1996) were kindly provided by Randy Moon. BMP4 in the vector pCS2+ (HemmatiBrivanlou and Thomsen, 1995) was a kind gift of Gerry Thomsen. The entire open reading frame of Xslug was amplified by low-cycle number PCR using PWO polymerase (Boehringer Mannheim) from stage $17 \mathrm{cDNA}$ and cloned into the vector $\mathrm{pCS} 2+$. Amplified products were sequenced on both strands using an automated DNA sequencer. One sequenced clone was identical to the previously published sequence. A second clone differed from the published sequence at five positions, but translated to an identical protein. Both clones performed similarly in overexpression experiments.

\section{Fixation and whole-mount in situ hybridization}

Embryos and explants were fixed for 1 hour in MEMFA, and stored dehydrated in $100 \%$ methanol. For $\beta$-galactosidase staining, embryos were washed $2 \times$ in PBS and $2 \times$ in staining solution $(10 \mathrm{mM}$ $\mathrm{K}_{3} \mathrm{Fe}(\mathrm{CN})_{6}, 10 \mathrm{mM} \mathrm{K}_{4} \mathrm{Fe}(\mathrm{CN})_{6}, 1 \mathrm{mM} \mathrm{MgCl}_{2}$ in PBS). Color reaction was carried out overnight at room temperature in staining solution containing $1.5 \mathrm{mg} / \mathrm{ml} \mathrm{X-gal.} \mathrm{In} \mathrm{situ} \mathrm{hybridization} \mathrm{was} \mathrm{carried} \mathrm{out} \mathrm{with}$ digoxigenin-labelled antisense RNA probes as described in Knecht et al. (1995). Alkaline phosphatase detection reactions were carried out using NBT/BCIP (Gibco BRL) as a substrate. Xslug (Mayor et al., 1995), Xtwi (Hopwood et al., 1989) and Xsox2 (Mayor et al., 1995) probes were kindly provided by Roberto Mayor, Peter Vize and Rob Grainger, respectively. Embryos and explants were photographed in $100 \%$ methanol. For histology, embryos and explants were incubated in Histisol for 30 minutes, embeded in paraplast and sectioned at 10$12 \mu \mathrm{m}$.

\section{Reverse transcription-PCR analysis}

RT-PCR analysis was carried out as previously described (LaBonne and Whitman, 1994). Serial dilutions of whole embryo cDNA were used to ensure the quantitative nature of the PCR reactions with each primer pair. PCR cycles were for 30 seconds at $94^{\circ} \mathrm{C}, 60$ seconds at $55-60^{\circ} \mathrm{C}$ and 30 seconds at $72^{\circ} \mathrm{C}$. Primer pairs utilised were as follows:

Xslug (Mayor et al., 1995)

U: CAATGCAAGAACTGTTCC

D: TCTAGGCAAGAATTGCTC

Xsna (Essex et al., 1993)

U: AAGCACAATGGACTCCTT

D: CCAATAGTGATACACACC

Xtwi (Hopwood et al., 1989)

U: AGTCCGATCTCAGTGAAGGGCA

D: TGTGTGTGGCCTGAGCTGTAG

Epidermal Keratin (Jonas et al., 1985)

U: CACCAGAACACAGAGTAC

D: CAACCTTCCCATCAACCA

NCAM (Kintner and Melton, 1987)

U: CACAGTTCCACCAAATGCCG

D: GGAATCAAGCGGTACAGA

EF1 $\alpha$ (Krieg et al., 1989)

U: CAGATTGGTGCTGGATATGC

D: ACTGCCTTGATGACTCCTAG

\section{RESULTS}

\section{Manipulation of BMP signaling in whole embryos}

To address whether graded amounts of BMP signaling play an instructive role in the specification of neural crest fates, we manipulated the levels of BMP signaling in whole embryos and assayed the effects on expression of an early neural crest 
marker, Xslug. In order to upregulate BMP signaling in the ectoderm, increasing amounts of BMP4 mRNA were microinjected into one dorsoanimal blastomere at the 8-cell stage. Embryos were cultured until stage 17 when they were harvested for in situ hybridization using a Xslug antisense probe. While a range of phenotypes was obtained with each injected dose, the average phenotype increased in severity with dose and a representative phenotype for each dose is presented in Fig. 1.

At the lowest dose (50 pg, $n=60)$, most embryos were mildly ventralized and Xslug expression was not markedly reduced. The two characteristic domains of Xslug expression in the neural folds of the hindbrain were deflected toward the midline (Fig. 1B), reflecting a loss of dorsoanterior character in the transverse neural folds. At higher doses (200 pg, $n=68$ ) increasing numbers of embryos were severely ventralized, exhibiting considerable microcephaly and Xslug expression domains were fused at the ventral aspect of markedly reduced head folds (Fig. 1C). This is likely due to an absence of forebrain and cement gland progenitors normally found in this region. Xslug expression was only lost at doses of BMP4 that eliminated the neural folds completely (1 ng, $n=40)$ (Fig. 1D). Thus, Xslug expression does not appear to be eliminated in response to increases in BMP4 levels, but rather continues to reflect the boundary between the diminishing neural plate and the surrounding non-neural ectoderm. Significantly, no dose of BMP4 was observed to increase neural crest formation at the expense of the neural plate, as might be expected if

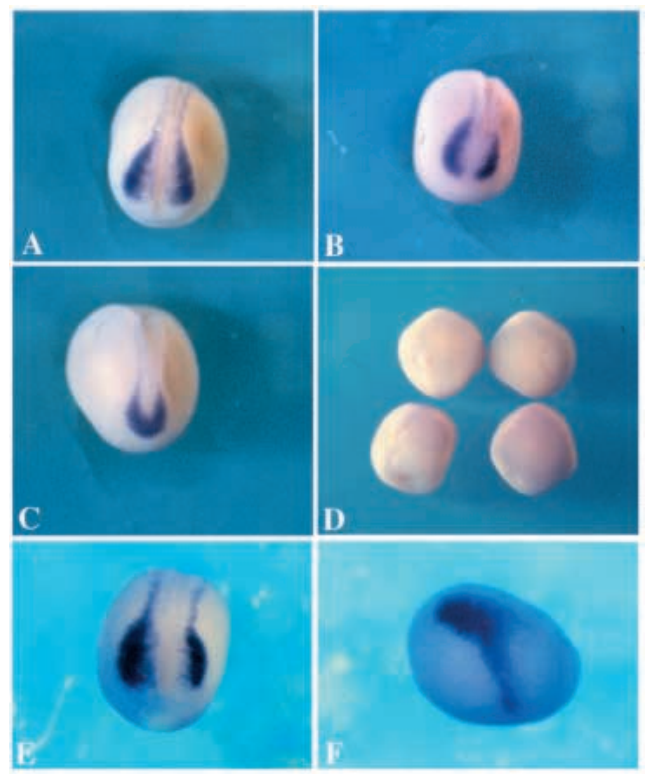

Fig. 1. Xslug expression is altered in response to changes in BMP activity. Whole-mount in situ hybridization showing Xslug expression in stage 17 control embryos (A) and in embryos injected in one cell at the 2-cell stage with $50 \mathrm{pg}$ (B), $200 \mathrm{pg}$ (C) or $1 \mathrm{ng}$ (D) BMP4 mRNA. With increasing BMP concentration Xslug expression domains become deflected toward the midline (B), fuse (C) and are lost in ventralized embryos lacking a discernible neural plate (D). (E,F) Xslug expression in embryos injected in one cell at the 2-cell stage with $200 \mathrm{pg}$ of chordin mRNA. Xslug expression is expanded laterally $(\mathrm{E})$ and/or ventrally $(\mathrm{F})$ on the injected side. intermediate BMP doses were primarily responsible for directing the prospective ectoderm toward neural crest fates.

To examine the effects of decreased BMP activity on Xslug expression, $200 \mathrm{pg}$ of chordin mRNA was injected into one cell of 2-cell-stage embryos. At stage 17, these embryos displayed increased levels of Xslug expression on the injected side. Ectopic Xslug expression was contiguous with endogenous expression domains and expansion was always into more lateral regions, at the expense of non-neural ectoderm, rather than into more medial neural plate regions (Fig. 1E). Ectopic Xslug expression was sometimes found in a band extending toward the ventral aspect of the embryo, as depicted in Fig. 1F. At doses of chordin that hyperdorsalized the embryo, Xslug expression was found in a band around the circumference of the radially symmetric embryo as has previously been described for noggin (Mayor et al., 1995; and not shown).

\section{Chordin overexpression in ectodermal explants}

It has recently been demonstrated that BMPs can specify distinct ectodermal cell fates, including epidermis, cement gland and neural plate, in a concentration-dependent manner (Wilson and Hemmati-Brivanlou, 1995; Wilson et al., 1997). Our observation that reductions in BMP activity following chordin overexpression lead to increases in Xslug expression suggested that specific levels of BMP signaling might play a role in the specification of neural crest cell fate. We therefore asked if chordin overexpression was sufficient to induce expression of neural crest markers in ectodermal explants. Chordin mRNA was injected in the animal poles of both cells of 2-cell embryos at doses ranging from $10 \mathrm{pg}$ to $2 \mathrm{ng}$. Animal

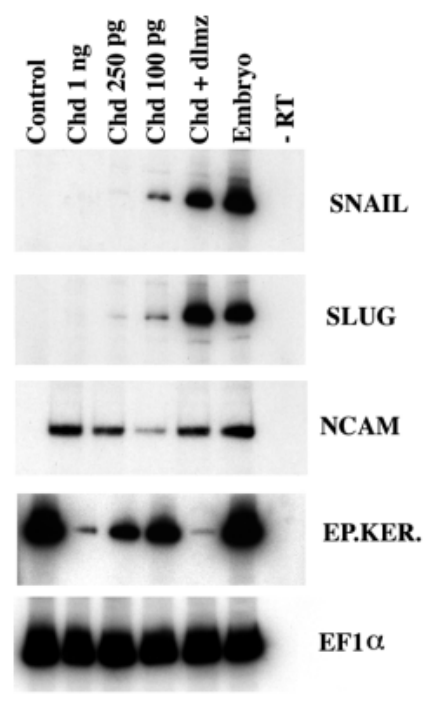

Fig. 2. Chordin induces low levels of neural crest gene expression in ectodermal explants. RT-PCR analysis of ectodermal explants from embryos injected with a range of concentrations (10 pg-2 ng) of chordin mRNA. Representative doses are shown. Explants were isolated at late stage 9 and harvested at stage 17. Control explants are from uninjected embryos. Whole embryos and chordin-expressing explants conjugated with dlmz serve as positive controls. All samples were analyzed with primers specific for the neural crest markers $X$ sna and Xslug, the pan-neural marker NCAM, the mesodermal marker muscle actin and epidermal keratin. EFI $\alpha$ serves as a loading control. 
caps were explanted at late stage 9 and cultured to stage 17 when they were harvested for RT-PCR analysis.

We found that high doses of chordin, which significantly inhibited the expression of epidermal keratin (Jonas et al., 1985 ) and induced expression of NCAM (Kintner and Melton, 1987), did not induce expression of early neural crest markers such as Xsna (Essex et al., 1993) and Xslug (500 pg-2 ng, Fig. 2 and not shown). At lower doses of chordin, however, low level expression of these markers could be induced (100-250 pg, Fig. 2 and not shown). Induction of neural crest markers in these samples could be the result of secondary interactions between chordin-induced neural and epidermal cell types, as these samples also expressed significant amounts of epidermal keratin. The levels of neural crest markers induced in these experiments were below that which could be detected by in situ hybridization. (Fig. 3B and not shown) No dose of chordin was found that induced neural crest markers in the absence of neural and epidermal markers, or which induced neural crest markers to levels consistent with their endogenous expression.
Similar results were obtained using either noggin or a dominant negative BMP receptor to inhibit BMP signaling (data not shown). This suggests that, while the regional level of BMP signaling in the ectoderm may play a role in the specification of the neural crest, this alone is insufficient to account for neural crest induction in vivo. Consistent with this, no dose of chordin was found that could induce melanocytes, a neural crest derivative, in ectodermal explants (not shown). In contrast, when chordin-expressing explants were conjugated with dorsolateral marginal zone tissue (dlmz, prospective somitic mesoderm), increased levels of neural crest markers were induced and melanocytes formed (Fig. 2 and not shown).

\section{A second signal is required for neural crest induction}

As chordin overexpression alone was insufficient to induce robust expression of neural crest markers, we asked what signal might be able to cooperate with chordin to induce neural crest. Recently, it has been demonstrated that FGF and Wnt family
Fig. 3. Chordin-expressing ectoderm can be lateralized to neural crest fates by members of the FGF and Wnt families. (A) Schematic of the assay. Conjugates were made by combining explants from control $(\mathrm{C})$ or chordin (CHD)-injected embryos with explants expressing XeFGF or XWnt-8 at late stage 9 . Explants were isolated at stage 17 for analysis by in situ hybridization or RT-PCR. (B) In situ hybridization showing Xslug expression in conjugates. Xslug expression is found in CHD/XWnt- 8 and $\mathrm{CHD} / \mathrm{XeFGF}$ conjugates but not in conjugates of control ectoderm with CHD, XeFGF or XWnt-8. (C) Section through a CHD/Wnt conjugate slug expression is found throughout the conjugate except for a crenelated epithelial-like outer layer. (D) In situ hybridization showing expression of the neural plate marker Xsox 2 in stage 17 embryos and in conjugates. $X$ sox 2 expression is found throughout $\mathrm{C} / \mathrm{CHD}$ conjugates, but is diminished in CHD/XWnt- 8 conjugates. (E) RT-PCR analysis of gene expression in conjugates. Controls and primers are the same as in Fig. 1. (F) Coexpression of a dominant inhibitory Wnt (dnWnt) inhibits the induction of neural crest markers in CHD/FGF conjugates.
A
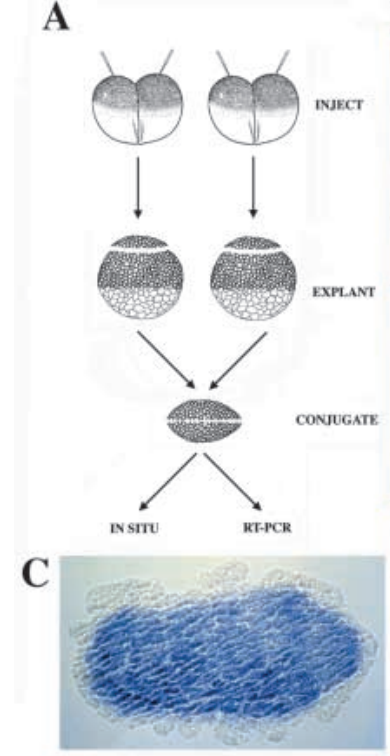

B

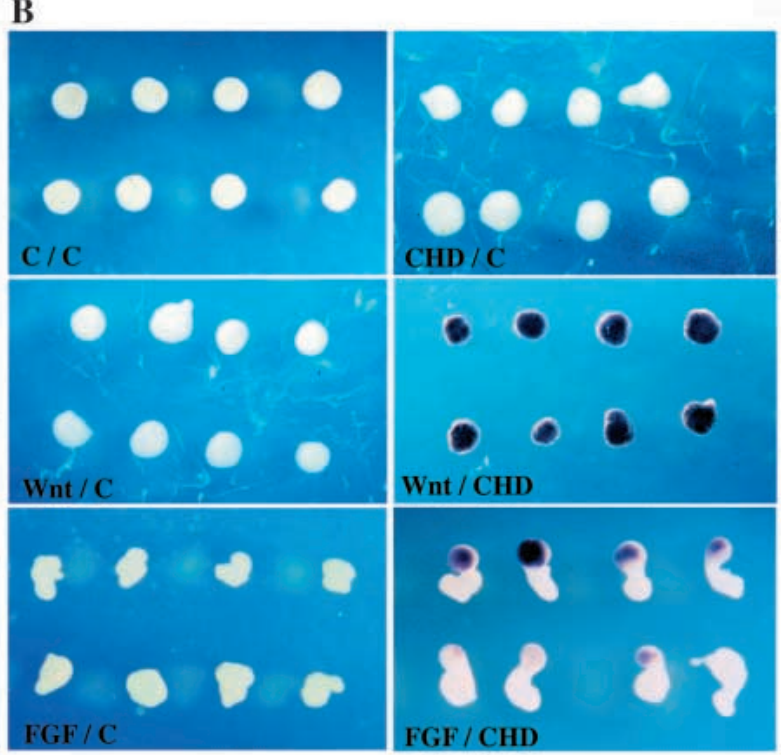

D

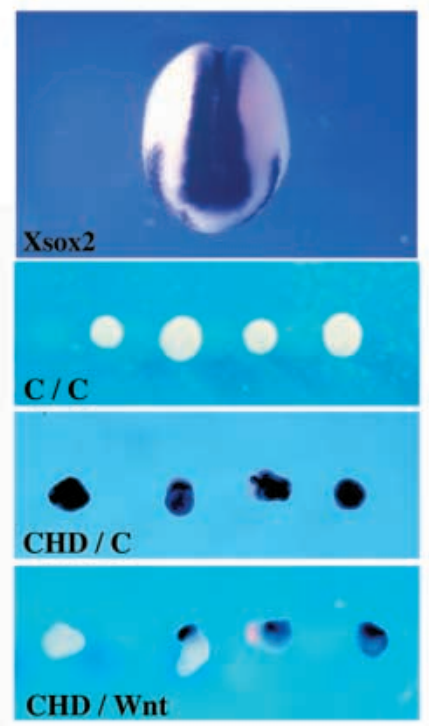

E

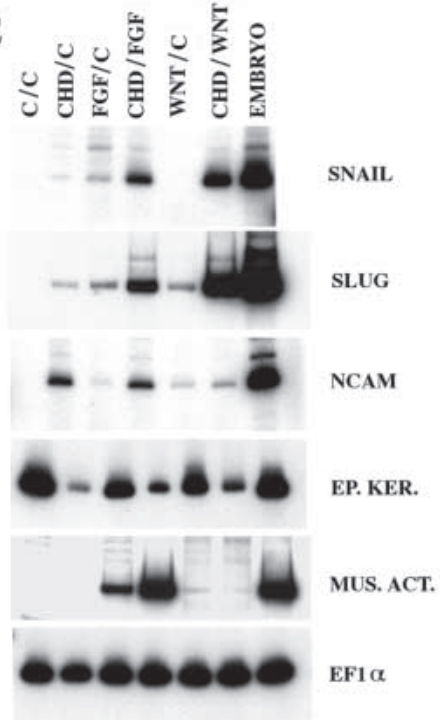

F

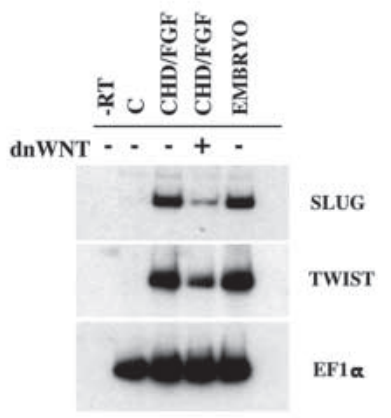




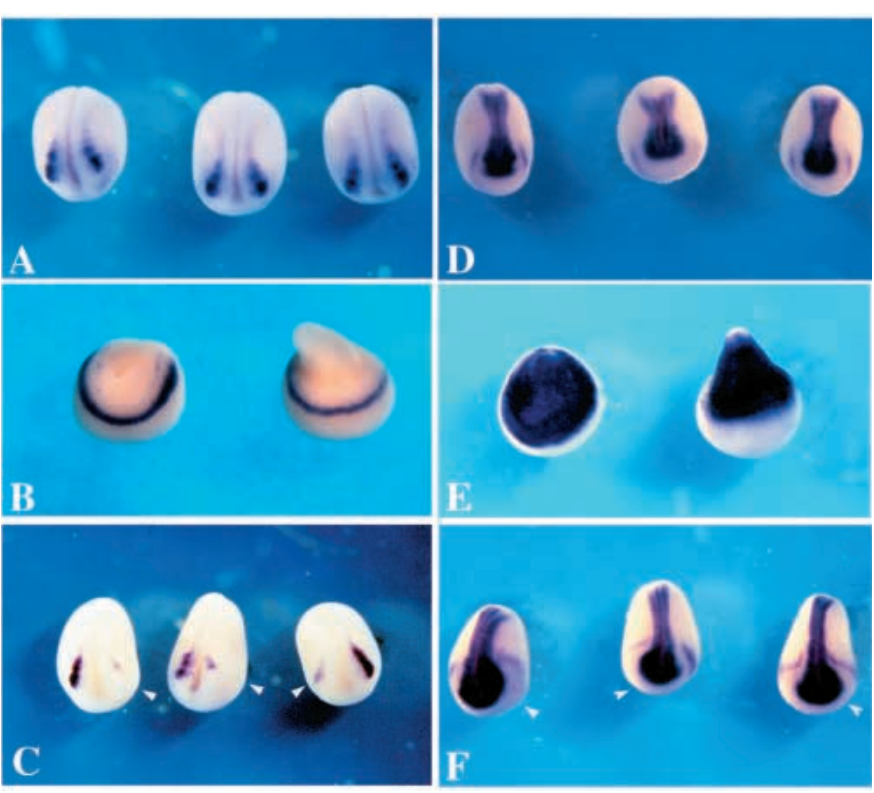

Fig. 4. Xslug expression is altered in response to changes in Wnt activity. In situ hybridization showing Xslug (A-C) and Xsox2 (D-F) expression in control embryos (A,D) or embryos injected in one cell at the 2-cell stage with $200 \mathrm{pg}$ XWnt- 8 mRNA (B,E) or $1 \mathrm{ng}$ of dominant negative XWnt- 8 mRNA (D,F). Arrowhead denotes the injected side of embryos in $\mathrm{C}$ and $\mathrm{F}$.

members can alter the anterioposterior character of neural tissue induced by noggin, another BMP antagonist (McGrew et al., 1995; Kengaku and Okamoto, 1993; Lamb and Harland, 1995; Cox and Hemmati-Brivanlou, 1995). We therefore asked whether these factors could alter the mediolateral character of neural tissue induced by chordin. To address this, we used an assay system which allows prospective neural tissue to be exposed to potential modifying signals at stages when neural crest induction is likely to be occurring in vivo (Fig. 3A). Control animal caps, or animal caps expressing eFGF or XWnt-8, were explanted at late stage 9 and conjugated with animal caps expressing chordin. These conjugates were cultured to stage 17 and assayed for neural crest gene expression by in situ hybridization or RT-PCR.

Conjugates composed of chordin-expressing ectoderm and control ectoderm did not express Xslug to levels detectable by in situ hybridization, consistent with a model in which inhibition of BMP signaling is insufficient to specify neural crest cell fates. When chordin-expressing explants were conjugated with explants expressing XWnt-8, however, significant levels of Xslug expression were induced (Fig. 3B). Similar results were obtained using conjugates of chordin and eFGF (Fig. 3B), confirming previous observations of neuralcrest-forming interactions between $\mathrm{bFGF}$ and noggin (Mayor et al., 1995). The observed Xslug induction could be secondary to mesoderm induced in eFGF-expressing explants, as these explants express muscle-specific actin (Fig. 3E). We found that XWnt-8 was a better inducer of Xslug than was eFGF, although the extent of induction by both was dose-dependent. As FGF is capable of inducing XWnt-8 expression in mesoderm induction assays, is seemed possible that the ability of FGF to induce neural crest markers in our conjugate assay was secondary to its ability to induce Wnt expression. To address this possibility, the ability of eFGF to cooperate with chordin to induce neural crest markers was assayed in the presence and the absence of a dominant inhibitory mutant of XWnt-8 that has been shown to inhibit XWnt-8 signaling in whole embryos (Hoppler et al., 1996). Co-expression of the dominant inhibitory Xwnt-8 significantly inhibited the ability of eFGF to induce neural crest markers in chordin-expressing ectoderm, suggesting that this ability may be indirect (Fig. 3F).

As neither chordin nor XWnt-8 are mesoderm-inducing factors, this induction appears to be mesoderm-independent. Consistent with this, no muscle actin expression was observed in these conjugates (Fig. 3E). Interestingly, chordin/XWnt-8 conjugates can give rise to solid blocks of Xslug-expressing tissue at stage 17 (Fig. 3B,C). As Xslug is expressed on both sides of these conjugates, this implies either that XWnt-8 is directly inducing Xslug expression in cells where BMP signaling has been inhibited by chordin, or alternatively, if the effects are indirect, that they are mediated by another soluble factor. Xnr3, a soluble TGF- $\beta$-related growth factor that can be induced by Xwnt- 8 does not induce neural crest markers in this assay (unpublished observation). Neither eFGF nor XWnt-8 alone induced Xslug to levels detectable by in situ hybridization, although low levels of expression could sometimes be detected by RT-PCR (Fig. 3E). Lateralization of chordin-induced neural tissue by XWnt- 8 was accompanied by loss of expression of the neural plate marker Xsox2 and resulted in the induction of significant numbers of melanocytes (Fig. 3D and not shown).
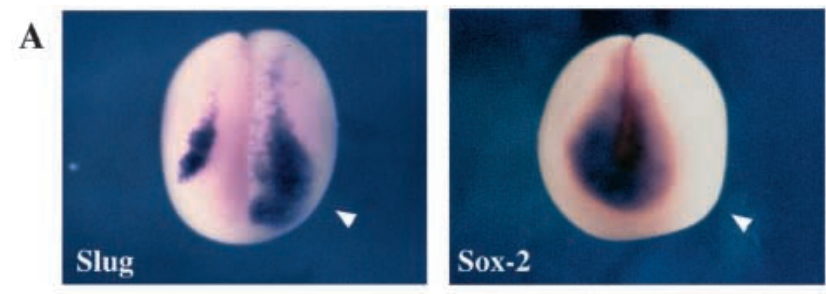

B
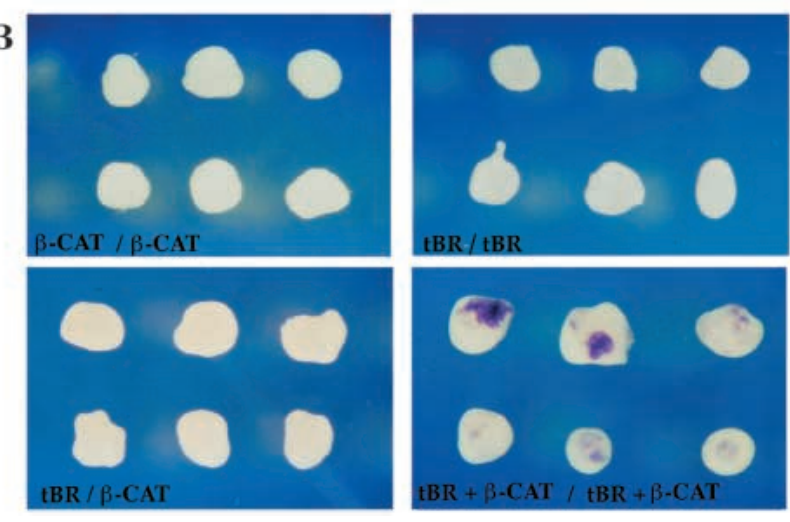

Fig. 5. Effects of $\beta$-catenin overexpression in whole embryos and conjugates. (A) in situ hybridization showing expression of Xslug and Xsox-2 in embryos injected in one cell at the 2-cell stage with $100 \mathrm{pg} \beta$-catenin. Expanded expression of the neural crest marker and diminished expression of the neural plate marker is seen on the injected side (white arrowheads). (B) In situ hybridization of conjugates which tBR and $\beta$-catenin were each expressed in only one half of each conjugate, or were coexpressed in both halves of each conjugate. Xslug expression is found only in conjugates in which tBR and $\beta$-catenin are coexpresed in the same cells. 
Fig. 6. The effects of Wnt signaling on neural crest induction are direct.

(A) Section through the midbrain region of a stage 18 embryo coinjected in one cell at stage 2 with $\beta$-catenin and $\beta$-galactosidase mRNAs. Embryos were stained for $\beta$ galactosidase activity (light blue) and processed for in situ hybridization using a Xslug probe (purple). Ectopic Xslug expression is restricted to cells expressing $\beta$-galactosidase. (B) Section through the rostral trunk region of a similarly treated embryo. $\beta$-galactosidase expression is seen exclusively in cells of ectodermal lineage. $\beta$-catenin overexpression induced ectopic Xslug expression lateral to endogenous expression domains, at the expense of epidermis. (C) Section through the caudal hindbrain region of a stage 16 embryo coinjected with dnWNT and $\beta$-galactosidase mRNAs. Expression of the injected mRNAs is exclusively ectodermal and mesodermal derivatives appear normal. (D) Closeup of a region of residual Xslug expression. Inhibition of Xslug expression was noted only in or directly adjacent to cells which expressed high levels of $\beta$-galactosidase activity.

\section{Changes in Wnt expression perturb neural crest induction}

As XWnt-8-expressing tissue can cooperate with chordinexpressing tissue to induce neural crest markers in vitro, we examined the effects of modulating Wnt activity in whole embryos. Embryos were injected in the animal pole of one cell at the 2-cell stage with $200 \mathrm{pg}$ XWnt-8 mRNA. Overexpression of XWnt-8 resulted in embryos $(n=43)$ that were hyperdorsalized to varying degrees. Xslug expression was found in a band around radially symmetric embryos, or partially around embryos retaining a recognizable neural plate (Fig. 4B). In both cases, Xslug expression appears to reflect the boundary of neural plate and non-neural ectoderm, as seen by comparing the expression patterns of Xslug and the neural plate marker Xsox2 (Fig. 4B versus E).

In order to determine if $\mathrm{Wnt}$ signaling is required for neural crest formation in vivo, we investigated the effects of decreasing Wnt activity in whole embryos. $1 \mathrm{ng}$ of dominant negative XWnt-8 mRNA was injected in the animal pole of one cell of 2-cell-stage embryos. A significant loss of Xslug expression was found on the injected side of these embryos, demonstrating a requirement for a Wnt signal for neural crest induction. (Fig. 4C). This loss of Xslug expression appears to be accompanied by a slight increase in the domain of Xsox2 expression (Fig. 4F).

\section{The effects of Wnt signaling on neural crest formation are direct}

The dorsalized phenotype of XWnt-8-injected embryos made it difficult to evaluate any direct effect of ectopically expressed XWnt-8 on Xslug expression. Although expression of Xslug was expanded, this was likely secondary to the expanded neural plates in these embryos. In order to more clearly investigate the effects of increased Wnt signaling on neural crest formation in vivo, we overexpressed $\beta$-catenin, a downstream mediator of Wnt signals (Fagotto et al., 1997). As $\beta$-catenin functions in the cytosol and nucleus during Wnt signaling, its effects should be cell autonomous. This makes it easier to control what cells receive ectopic Wnt signals and assess whether any observed effects on neural crest formation are direct or indirect.
$100 \mathrm{pg} \beta$-catenin mRNA was injected into one cell of 2-cell embryos. At stage 17, these embryos were harvested for in situ hybridization using probes for Xslug and Xsox-2. $\beta$-catenin overexpression resulted in a dramatic increase in Xslug expression on the injected side. (Fig. 5A) Similar to what was observed for chordin overexpression, the neural-crest-forming regions were expanded into more lateral and posterior regions of the prospective ectoderm. This was accompanied by a

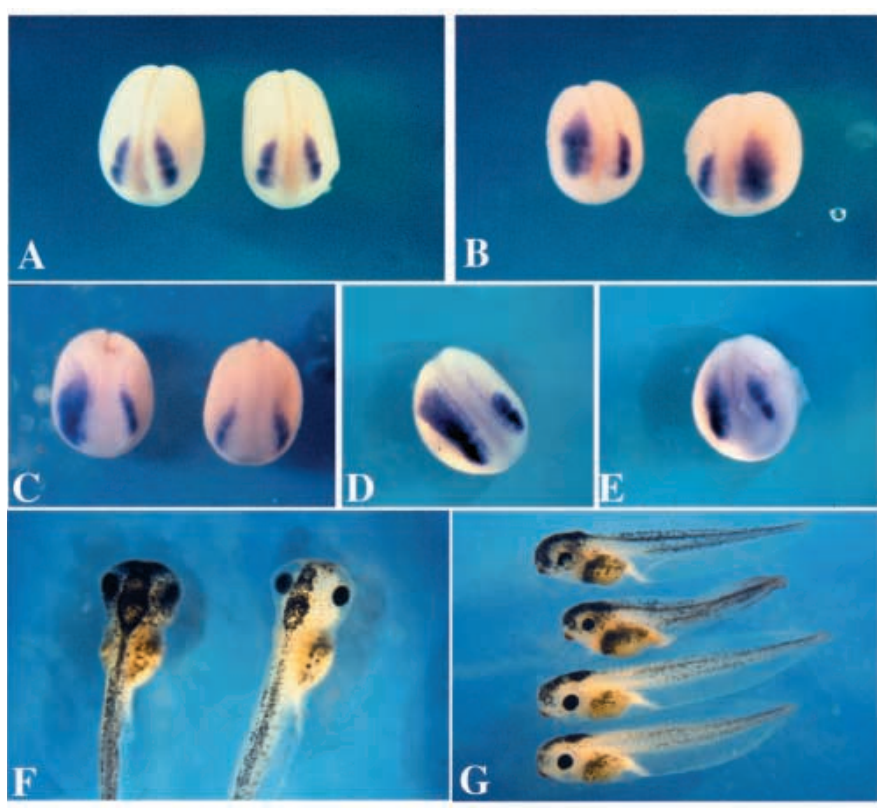

Fig. 7. Effects of Slug overexpression in whole embryos. In situ hybridization showing expression of Xtwi $(\mathrm{A}, \mathrm{B})$ and Xslug $(\mathrm{C}-\mathrm{E})$ in control embryos (A, both embryos; $\mathrm{C}$, right embryo) and embryos injected in one cell at the 2-cell stage with $0.5 \mathrm{ng}$ Slug mRNA. Expanded expression of neural crest markers is seen on the injected side. Increased numbers of melanocytes are found when such embryos are allowed to develop until stage 41. (F) Left embryo is injected, right embryo is a sibling control. (G) Top two embryos are injected, bottom two are sibling controls. 


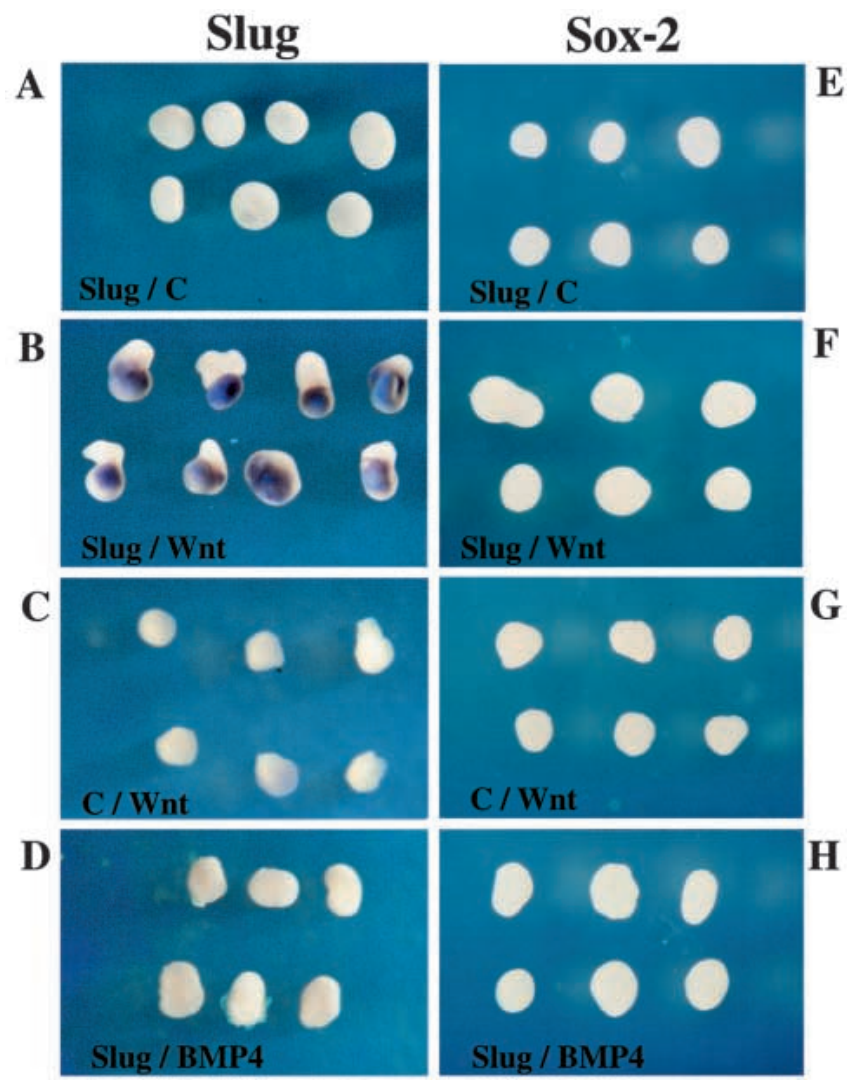

Fig. 8. Neural crest markers, but not neural markers, are induced in Slug/Wnt conjugates. (A-D) In situ hybridization showing expression of Xslug in conjugates of control or Slug-expressing explants with explants expressing Xwnt-8 or BMP4. Expression of Xslug is seen only in Slug-expressing explants exposed to a Wnt signal. (E-H) $X$ sox-2 expression is not seen in Slug-expressing conjugates.

decrease in the levels of Xsox-2 expression on the injected side. A more dramatic effect on the Xsox-2 expression domain was not necessarily expected as the expression patterns of Xsox-2 and Xslug are overlapping in vivo (unpublished observation.)

As $\beta$-catenin overexpression resulted in an expansion of neural-crest-forming regions, and as it acts in a cell autonomous fashion, it provides a tool for determining whether the effects of Wnt signaling on neural crest formation in vitro are direct or indirect. In these experiments, a dominant inhibitory BMP receptor (tBR) was used in place of chordin as the means of inhibiting BMP signaling (Graff et al., 1994). This allowed us to set up an assay in which the modulation of both Wnt and BMP signals is cell autonomous. Conjugate assays were set up in which tBR and $\beta$-catenin were each expressed in only one half of each conjugate, or were coexpressed in both halves of each conjugate. The conjugates were cultured until stage 17 and harvested for in situ hybridization. Significant Xslug expression was detected in conjugates in which tBR and $\beta$-catenin were coexpressed, but not in conjugates in which their expression was nonoverlapping (Fig. 5B). This strongly suggests that the effects of both molecules on neural crest formation are direct. It should be noted that high doses of $\beta$-catenin were capable of inducing Xslug expression in the absence of tBR-mediated BMP inhibition at late blastula stages. The levels of $\beta$-catenin mRNA

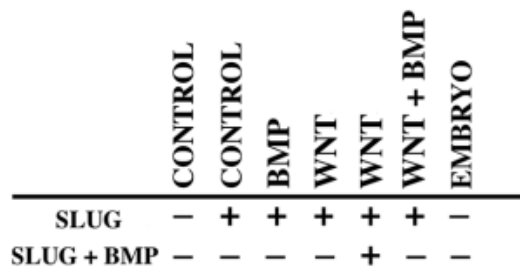

SNAIL

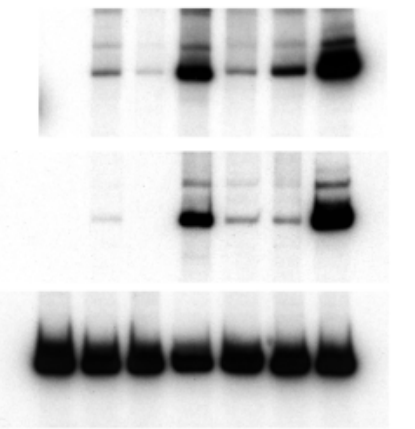

SLUG

TWIST

$\operatorname{EF} 1 \alpha$

Fig. 9. Neural crest induction in Slug-expressing conjugates is BMPsensitive. RT-PCR analysis of gene expression in Slug-expressing conjugates in the presence and absence of increased BMP levels. BMP is co-expressed in either the Slug-expressing or Wntexpressing halves of the conjugate. Xtwi is a neural crest marker. Other primers and controls are the same as in Fig. 1.

injected for conjugate assays was well below that required for tBR-independent Xslug induction (Fig. 5B and not shown.) These low levels of mRNA, together with the cell-autonomous nature of both tBR and $\beta$-catenin, most likely explain the rather patchy Xslug induction achieved in these experiments.

Since the effects of Wnt signaling on neural crest induction in vitro appeared to be direct, we next wished to determine whether the effects on neural crest formation in vivo were also direct. $100 \mathrm{pg} \beta$-catenin mRNA or $250 \mathrm{pg}$ dnWNT mRNA was coinjected with $\beta$-galactosidase mRNA in one cell of 2-cell embryos. Embryos were cultured to neurula stages, stained for $\beta$-galactosidase activity and processed for in situ hybridization using a Xslug probe. The embryos were then sectioned to determine the extent of overlap between the $\beta$-galactosidaseexpressing cells and the effects on Xslug expression. The effects of $\beta$-catenin overexpression on ectopic neural crest formation were found to be highly cell autonomous. Ectopic Xslug expression appeared to be restricted to cells expressing the injected mRNAs (Fig. 6A,B). $\beta$-galactosidase expression was often found exclusively in cells of ectodermal lineage, ruling out secondary effects from the mesoderm. Interestingly, $\beta$-catenin overexpression induced ectopic Xslug expression lateral and posterior to endogenous expression domains, largely at the expense of epidermis. This is similar to the phenotype observed following chordin overexpression. When the injected mRNA was inherited by cells in more medial regions of the neural plate, ectopic Xslug expression was not observed (Fig. 6A and not shown).

Similar to what was found for $\beta$-catenin overexpression, the effects of dnWnt overexpression on neural crest formation was 
observed to be highly cell autonomous. Inhibition of Xslug expression was noted only in or directly adjacent to cells that expressed high levels of $\beta$-galactosidase activity (Fig. 6C,D). Where there was residual slug expression, little or no $\beta$ galactosidase activity was observed (Fig. 6D). Expression of the injected mRNAs was often exclusively ectodermal; mesodermal derivatives appeared to develop normally in these embryos. Thus the inhibition of Xslug expression appears to reflect a requirement for Wnt signaling in the ectoderm fated to give rise to neural crest and is not a secondary consequence of defects in the underlying mesoderm.

\section{Role of the transcription factor Slug}

The above experiments suggest a model for neural crest induction in which inhibiting BMP signaling specifies a tissue that expresses very low levels of early neural crest markers, and which is competent to respond to lateralizing signals that enhance and maintain neural crest gene expression. Members of the Wnt and FGF family are sufficient to provide such a lateralizing signal, although the ability of FGF to do so may be indirect. We wished to know whether, once robust levels of early neural crest markers had been induced, subsequent neural crest ontogeny required either continued inhibition of BMP signals or the presence of a lateralizing signal.

To address this question, we assayed the effects of overexpressing the zinc finger transcription factor Slug in whole embryos and explants. We chose to overexpress Slug as it is one of the earliest neural crest markers, and as up until stage 17 it is expressed exclusively in the prospective neural crest. This is in contrast to other early neural crest markers such as Snail (Xsna) (Essex et al., 1993), a related zinc finger transcription factor, and Twist (Xtwi) (Hopwood et al., 1989), a helix-loop-helix containing factor, which are also expressed in the mesoderm at early stages. mRNA containing the coding region of Slug was injected in the animal pole of one cell of 2-cell-stage embryos. The embryos were cultured to stage 17 and processed for in situ hybridization using antisense probes for Xtwi or for the 3' UTR of Xslug. The latter probe will recognize endogenous Xslug, but not the injected Slug message.

The external morphology of injected embryos was fairly normal through swimming tadpole stages, although headfolds were sometimes slightly enlarged on the injected side at neurula stages. In situ hybridization demonstrated that at these stages, expression of Xtwi (Fig. 7B) and Xslug (Fig. 7C-E) was enhanced on the injected side of the embryo. Similar to what was seen following chordin or $\beta$-catenin overexpression, the expression of neural crest markers was expanded laterally at the expense of non-neural ectoderm, rather than into the neural plate proper (Fig. 7B-E). Expression of neural crest markers was sometimes enhanced posteriorly on the injected side, extending into posterior hindbrain and spinal cord regions where neural crest induction is generally less massive (Fig. 7D). Expression of neural crest markers was increased to some degree in $87 \%$ of injected embryos $(n=130)$. The observation that Slug can enhance its own expression, in addition to that of later neural crest markers such as Xtwi, suggests that endogenous Slug may play a role in the maintenance of its own expression.

In order to examine further the effects of Slug overexpression, some injected embryos were allowed to develop to swimming tadpole stages, when they were scored for quantitative or spatial changes in the formation of neuralcrest-derived melanocytes. Slug-injected embryos $(n=91)$ displayed a pronounced increase in the number of melanocytes formed relative to control embryos ( $n=102$, Fig. 7F,G). These supernumerary melanocytes had a normal dendritic morphology, were light-responsive and populated characteristic regions of the embryo, albeit in larger numbers. Injection of $\beta$-galactosidase mRNA caused no noticeable increase in the number of melanaocytes relative to uninjected embryos. These results indicate that Slug overexpression leads not only to increased expression of early neural crest markers, but also to increased numbers of at least one neural crest derivative.

\section{Slug overexpression in ectodermal explants}

The observation that, following Slug overexpression, ectopic expression of neural crest markers is limited to areas contiguous with endogenous neural-crest-forming regions suggested that Slug expression itself was insufficient to direct a program of neural crest ontogeny. Instead, it appears that neural crest formation additionally requires signals present either laterally, in the non-neural ectoderm, or in the underlying mesoderm.

In order to examine further the neural-crest-forming potential of Slug-expressing ectoderm, we performed explant experiments similar to those described in Fig. 3. Slug mRNA was injected into the animal poles of 2-cell-stage embryos, and animal pole explants were isolated at late blastula stages and cultured until stage 17. Slug-expressing explants cultured in isolation, or conjugated with control explants, failed to express endogenous Xslug or Xtwi to levels detectable by in situ hybridization (Fig. 8A and not shown) although, when explants were isolated at early gastrula stages, small foci of faint Xslug expression were sometimes noted (not shown). These data suggested that Slug overexpression itself is insufficient to elicit neural crest gene expression. We next asked if XWnt-8, which is sufficient to lateralize chordin-induced neural tissue to neural crest fates, could also induce neural crest gene expression in Slug-expressing ectoderm. Neural crest-specific markers could be induced in all or most of the Slug-expressing cells in Slug/XWnt-8 conjugates but not in the Wnt-expressing cells (Fig. 8B), indicating that, unlike chordin, the effects of Slug on neural crest induction were cell-autonomous. eFGF was able to induce expression of neural crest markers in Slugexpressing ectoderm, although it was a less potent inducer than XWnt-8 (not shown). BMP4 was unable to induce neural crest markers in Slug-expressing ectoderm (Fig. 8D). Induction of neural crest markers in Slug-expressing ectoderm was not dependent upon the expression of neural plate markers such as Xsox-2 (Fig. 8E-H).

In order to analyse more sensitively the ability of Slug to induce neural crest gene expression, similar experiments were carried out and analyzed by RT-PCR. In these experiments, Slug overexpression was sufficient to induce very low level expression of Xslug itself and of Xtwi. The significance of this induction, which appears to be below the level detectable by in situ hybridization, is unclear. It is reminiscent, however, of the low-level expression of these markers noted following chordin overexpression. Slug overexpression does not appear to induce any detectable expression of Xsna, which is 
expressed prior to Xslug in whole embryos. Similarly, both Slug/XWnt-8 conjugates and Slug/eFGF conjugates expressed robust levels of Xslug and Xtwi, but expressed Xsna weakly if at all (Fig. 9).

If neural crest induction requires both a suppression of BMP-mediated epidermal fate, together with a lateralizing signal that can be provided by XWnt- 8 or eFGF, the above experiments suggest that Slug overexpression is sufficient to bypass the need to inhibit BMP signaling. We therefore reasoned that neural crest induction in Slug/XWnt-8 conjugates should be insensitive to changes in BMP levels. To test this, BMP4 mRNA was co-expressed in either the Slug or XWnt-8-expressing portions of such conjugates and the effects on neural crest marker expression was assayed by RT-PCR. Surprisingly, BMP4 expression was able to repress expression of Xtwi and Xslug in these conjugates (Fig. 9), indicating that some aspect of this process remains sensitive to increases in BMP signaling.

\section{DISCUSSION}

\section{BMP as a morphogen}

Accumulating evidence supports the idea that, at least in Xenopus, BMPs mediate a choice between neural and epidermal fates in the prospective ectoderm (Wilson and Hemmati-Brivanlou, 1995; Piccolo et al., 1996; Zimmerman et al., 1996). More recently, our knowledge of the concentrationdependent patterning abilities of BMP4 has been expanded to include induction of the cement gland (Wilson et al., 1997), a highly specialized tissue that is induced at the anterior-most junction of neural and non-neural ectoderm. Indeed, as has been found in Drosophila (Ferguson and Anderson, 1992), BMP4 appears to be capable of acting as a morphogen, evoking distinct cell fate responses at different concentrations. A simple model for induction of the neural crest, a cell type that also arises at the junction between neural and non-neural ectoderm, would be that, like cement gland, neural crest is specified at levels of BMP signaling intermediate to those that induce neural plate and epidermis.

Our experiments using chordin as a BMP antagonist suggest, however, that this model is overly simplistic. We find no level of chordin overexpression capable of inducing early neural crest markers to levels consistent with their expression in vivo, although it remains possible that other neural inducers may be capable of doing so (Morgan and Sargent, 1997). Indeed, under a model in which the absolute level of BMP signaling was the dominant factor influencing the formation of neural crest, we would expect that, if the BMP concentration was raised locally, on one side of the prospective ectoderm, neural crest formation would tend to be lost on that side of the embryo. Instead, while such a local increase in BMP levels seems to lead to a reduction in the size of the neural plate, the neural crest itself is, to a surprising degree, unaffected. Expression of neural crest markers continues at the border between the prospective epidermis and the diminishing neural plate until BMP levels are achieved that eliminate the neural plate altogether. These experiments suggest that it is the border region itself that is critical for neural crest formation, rather than the absolute level of BMP signaling at the border. One caveat to these findings is that the timing of the BMP signal may be crucial to realizing its effect. BMP signaling levels do appear to play an important role in the formation of the border region, as seen by the expanded expression of neural crest markers following chordin overexpression.

\section{Lateralizing versus posteriorizing influences}

If a distinct level of BMP signaling is by itself insufficient for neural crest formation, what additional signals are required? Recent work addressing the anterior-posterior patterning of the neural plate demonstrates that members of the FGF and Wnt families of growth factors can caudalize or 'transform' prospective forebrain tissue to hindbrain and spinal cord fates (McGrew et al., 1995; Kengaku and Okamoto, 1993; Lamb and Harland, 1995; Cox and Hemmati-Brivanlou, 1995). This transforming activity was first suggested by the experiments of Nieuwkoop (Nieuwkoop and Albers, 1990). It appears that these same families of growth factors, as demonstrated here using XWnt-8 and eFGF, are able to lateralize prospective neural tissue as well, inducing neural crest formation. Alternatively, these factors may be exerting a posteriorizing influence, not on anterior neural plate, but rather on anterior border cell types such as cement gland.

The latter model might explain the varying degrees of neural crest induction that can be achieved in chordin/Wnt conjugates. Recently, it has been demonstrated that, at some concentrations of the BMP antagonist noggin, ectodermal explants are induced to express multiple neural plate markers, with some degree of dorsoventral pattern (Knecht and Harland, 1997). In addition, some concentrations of noggin will induce predominantly neural plate markers or predominantly cement gland markers (Wilson et al., 1997). If only prospective cement gland can be posteriorized to neural crest fates, then the degree of cement gland induction at a particular dose of BMP antagonist would limit the degree of neural crest formation in chordin/Wnt conjugates. Experiments are currently underway to address this possibility. Two experiments in the current study argue against this model, however. In the two halves of chordin/Wnt conjugates there is likely to be a gradation in the degree of BMP inhibition, with lower levels of BMP activity found on the chordin-expressing side. Nevertheless, induction of neural crest markers can be achieved throughout the conjugate. In addition, in mildly ventralized embryos, anteriormost fates such as cement gland are among the first to be lost. If only this anterior border cell type can be posteriorized to neural crest fates, one might expect neural crest cells to be absent in such embryos. Expression of neural crest markers is not lost in these embryos, however, but instead continues to reflect the boundary between neural plate and epidermis.

\section{Role of Wnts and FGFs in neural crest induction}

The importance of a Wnt signal for neural crest formation is demonstrated by the loss of expression of neural crest markers following overexpression of a dominant negative XWnt-8. Recently, it has also been demonstrated that overexpression of a dominant inhibitory FGF receptor has a similar effect (Mayor et al., 1997). As ventrolateral mesoderm is a potent neural crest inducer, and as dnWnt has been shown to inhibit somite formation when expressed in the mesoderm, it was possible that the effects of Wnt inhibition on the neural crest were secondary to effects on the mesoderm. However, as a dramatic 
inhibition of neural crest induction is noted in embryos in which dnWNT is expressed exclusively in the ectoderm, it appears that the requirement for Wnt signaling in the neural crest is independent of any role it may also play in the mesoderm. Consistent with this finding, when Wnt signaling is increased by overexpressing $\beta$-catenin, the effects on ectopic $X$ slug expression are cell autonomous and largely confined to more lateral and posterior region of the ectoderm.

As Wnt signaling has also been implicated in the posteriorization of anterior neural tissue (McGrew et al., 1995), it was possible that the effects of Wnt signaling on neural crest formation could be secondary to this posteriorization, and thus indirect. Several observations suggest that this is not the case, however. Wnt signaling can induce neural crest markers in Slug-expressing ectoderm. As Xslug is never expressed in anterior neural tissue and as Slug overexpression does not induce expression of neural plate markers such as Xsox-2, the neural-crest-inducing abilities of Wnt in this assay must be independent of its ability to posteriorize the neurectoderm. In addition, the ability of Wnt signals to cooperate with weak neuralizing signals to induce neural crest in ectodermal conjugates is cell-autonomous, and thus reflects a direct requirement for a Wnt signal during neural crest formation.

Consistent with this finding, the effects of up- or downregulating Wnt signaling on neural crest formation in vivo is also highly cell-autonomous. In addition, the ability of $\beta$ catenin overexpression to induce ectopic Xslug expression in cells normally fated to become epidermis is not consistent with a model in which the effects of Wnt signaling on the neural crest are secondary to the posteriorization of anterior neural tissue. Indeed, this result suggests that formation of the boundary between the neural and non-neural ectoderm is highly dynamic. Experiments are currently underway to address the mechanisms underlying the regulation of this boundary formation.

Are members of the FGF and Wnt families present at a time and place consistent with a role in this process? At gastrula stages, eFGF and XWnt- 8 are present in the ventral and lateral marginal zone, and could participate in early planar patterning events. Xsna expression, the earliest known marker of neural crest induction, can be detected by in situ hybridization at late gastrula stages (Essex et al., 1993), and it has recently been demonstrated that its expression requires only planar inductive signals (Poznanski et al., 1987). In addition, FGF receptordependent MAP kinase activity can be detected in the prospective ectoderm throughout gastrulation (LaBonne and Whitman, 1997). Wnt-1 and Wnt-3a are expressed in the dorsal neural tube of chick and mouse embryos, making them attractive candidates for a role in this process. Their expression is preceded by that of Slug, however, precluding a role for these Wnts in the earliest aspects of the inductive process. Interestingly, a recent murine Wnt-1/Wnt-3a double knockout displays a marked deficiency in neural crest derivatives (Ikeya et al., 1997), indicating that these molecules are most likely required for the maintenance and proliferation of the neural crest in the mouse. Recently, expression of Xenopus Wnt-3a has been described at the lateral edges of the open neural plate (McGrew et al., 1997). This raises the possibility that Wnt3A may be involved in earlier steps of neural crest induction in this system. Wnt 3A has been shown to induce expression of neural crest markers in noggin-neuralized ectoderm (Saint-Jennet et al., 1997). In addition, a novel Wnt family member, XWnt7B is present throughout the ectoderm during neural-crest-forming stages and could also play a role in this process (Chang and Hemmati-Brivanlou, 1998). Two avian Wnts are also present in the ectoderm bounding the open neural plate (C. Marcelle and M. B.-F., unpublished data). The role of these Wnts in neural crest induction can be addressed once their Xenopus homologues have been cloned.

\section{Role of the transcription factor Slug}

Expression of the zinc finger transcription factor Slug in the neural crest was first described in avian embryos, where it is expressed in the closing neural folds prior to the onset of neural crest migration (Nieto et al., 1994). Antisense experiments suggested that Slug may play a role in the migratory ability of the neural crest (Nieto et al., 1994). More recently, it has been demonstrated that Slug expression can promote migratory ability in other cell types (Savagne et al., 1997). In contrast, the neural crest-specific expression of Xslug commences at late gastrula stages (Mayor et al., 1995), long before any role in neural crest migration would dictate. The earliest migration of neural crest in Xenopus, in the prospective hindbrain region, does not occur until stage 19 when the neural tube has closed. The experiments described here suggest that Xslug, in addition to any role in migratory ability, also plays a role in earlier aspects of neural crest formation. Although Slug overexpression alone is insufficient to direct neural crest formation in ectodermal explants, it can do so in cooperation with a Wnt or FGF signal. Similarly, Slug overexpression causes an expansion of the neural crest domain and an increase in melanocyte formation in whole embryos. The inability of Slug overexpression to elicit such responses in ectodermal explants suggests either that Slug must act in cooperation with other transcriptional regulators, or that Slug requires posttranslational modification in order to effect changes in gene expression. It is interesting to note that Slug may play a role in regulating its own expression. Such an autoregulatory loop could be an important component in the maintenance of neural crest cell fate from its specification at late gastrula stages until migration commences at late neurula stages.

\section{Neural crest induction in avians and amphibians}

Several lines of evidence suggest, at first glance, that key aspects of neural crest induction may be different in avian and amphibian embryos. Why, for example, does expression of Slug commence at late gastrula stages in Xenopus, but not until the neural folds are closing in avian embryos? Does this reflect differing roles for Slug in the two systems, or a later specification of neural crest cell fate in avian embryos? It is possible that differences in the timing of Slug expression in avian versus amphibian embryos reflect a difference in the time that prospective neural plate/dorsal neural tube is exposed to the second lateralizing (or in the case of avian embryos, dorsalizing) signal. One possible reason why neural crest may be specified earlier in Xenopus is the different behavior of hindbrain neural crest in these embryos. In Xenopus, the cranial neural crest masses segregate from the neural tube prior to its closure and never participate in the movements of neurulation (Schroeder, 1970; Hall and Hörstadius, 1988). In contrast, the neural crest does not emerge from the dorsal neural tube of avian embryos until after neural tube closure (Hall and 
Hörstadius, 1988). Indeed, it has been demonstrated that until this time, single avian dorsal neural tube cells can give rise to epidermal, neural tube and neural crest derivatives (Selleck and Bronner-Fraser, 1995).

A second apparent difference between the two systems involves the requirement for a second, non-BMP signal during neural crest induction in Xenopus. Upon closer examination, however, this may prove to be true in avians as well. In both avian and amphibian embryos, a signal derived from the epidermis is sufficient to lateralize/dorsalize prospective neural tissue and evoke neural crest cell formation. The ability of BMPs to mimic this signal and induce neural crest from avian neural plate explants seems, at first glance, to preclude the need for additional modifying signals. It is possible, however, that the induction of neural crest in these explants is indirect. BMP treatment may result in the epidermalizing of a portion of the neural plate explant. This newly induced epidermis may then lateralize/dorsalize the remaining neural tissue, generating neural crest.

The current work suggests that, at least in Xenopus, endogenous BMP signaling must initially be inhibited to levels that can specify the neural plate border region; this tissue is then competent to respond to Wnt-like lateralizing signals that enhance and maintain neural crest induction. It remains possible that these interactions at the neural plate border lead to a subsequent upregulation of BMP activity in this region, and that this upregulation is also required for neural crest formation. Interestingly, expression of BMP4 and BMP7 is upregulated at the neural plate border region in Хеnopus (Hemmati-Brivanlou and Thomsen, 1995). The necessity of this upregulation for neural crest formation is currently being investigated.

The authors gratefully acknowledge A. Collazo, A. Knecht, C. Baker, S. Fraser and T. Moreno for critical reading of the manuscript; A. Gross and members of the laboratory for helpful comments and suggestions; and Eddy DeRobertis, Randy Moon, Rob Grainger, Gerry Thompsen, Jonathan Slack, Robert Davis, Peter Vize, Doug Melton and Roberto Mayor for providing reagents. C. L. is a fellow of the American Cancer Society. This work was also supported by USPHS NS36585 and NS34671 to M. B. F.

\section{REFERENCES}

Chang, C. and Hemmati-Brivanlou, A. (1998). Neural crest induction by Xwnt7B in Xenopus. Dev. Biol. 194, 129-134.

Christian, J. L., McMahon, J. A., McMahon, A. P. and Moon, R. T. (1991). XWnt-8, a Xenopus Wnt-1/int-1-related gene responsive to mesoderminducing growth factors, may play a role in ventral mesodermal patterning during embryogenesis. Development 111, 1045-1055.

Cox, W. G. and Hemmati-Brivanlou, A. (1995). Caudalization of neural fate by tissue recombination and bFGF. Development 121, 4349-4358.

Dickinson, M. E., Selleck, M. A. J., McMahon, A. P. and Bronner-Fraser, M. (1995). Dorsalization of the neural tube by the non-neural ectoderm. Development 121, 2099-2106.

Essex, L. J., Mayor, R. and Sargent, M. G. (1993). Expression of Xenopus Snail in mesoderm and prospective neural fold ectoderm. Dev. Dyn. 198, 108-122.

Fagotto, F., Guger, K. and Gumbiner, B. M. (1997). Induction of the primary dorsalizing center in Xenopus by the Wnt/GSK/beta-catenin signaling pathway, but not by Vg1, Activin or Noggin. Development 124, 453-460.

Fainsod, A., Deissler, K., Yelin, R., Marom, K., Epstein, M., Pillemer, G., Steinbeisser, H. and Blum, M. (1997). The Dorsalizing and neural inducing gene follistatin is an antagonist of BMP4. Mech. Dev. 63, 39-50.
Ferguson, E. L. and Anderson, K. (1992). decapentaplegic acts as a morphogen to organize dorso-ventral patterning in the Drosophila embryo. Cell 71, 451-461.

Graff, J. M., Thies R. S., Song,J. J., Celeste, A. J. and Melton, D. A. (1994) Studies with a Xenopus BMP receptor suggest that ventral mesoderminducing signals override dorsal signals in vivo. Cell 79,169-179.

Hall, B. K. and Hörstadius, S. (1988). The Neural Crest. Oxford: Oxford University Press.

Hemmati-Brivanlou, A., Kelly, O. G. and Melton, D. A. (1994). Follistatin, an antagonist of activin is expressed in the Spemann organizer and displays direct neuralizing activity. Cell 77, 283-295.

Hemmati-Brivanlou, A. and Thomsen, G. (1995). Ventral mesodermal patterning in Xenopus embryos: expression patterns and activities of BMP2 and BMP-4. Dev Genet 17, 78-89.

Hoppler, S., Brown, J. D. and Moon, R. T. (1996). Expression of a dominantnegative Wnt blocks induction of Myo-D in Xenopus embryos. Genes Dev. 10, 2805-2817.

Hopwood, N. D., Pluck, A. and Gurdon, J. B. (1989). A Xenopus mRNA related to Drosophila twist is expressed in response to induction in the mesoderm and the neural crest. Cell 59, 893-903.

Ikeya, M., Lee, S. M., Johnson, J. E., McMahon, A. P. and Takada, S. (1997) Wnt signaling required for expansion of neural crest and CNS progenitors. Nature, 389, 966-970.

Isaacs, H. V., Tannahill, D. and Slack, J. M. W. (1992). Expression of a novel FGF in the Xenopus embryo. A New candidate inducing factor for mesoderm formation and anteroposterior specification. Development 114, 711-720.

Jonas, E., Sargent, T. D. and Dawid, I. B. (1985). Epidermal keratin gene expressed in embryos of Xenopus laevis. Proc. Natl. Acad. Sci. 82, 54135417.

Kengaku, M. and Okamoto, H. (1993). Basic fibroblast growth factor induces differentiation of neural tube and neural crest lineages of cultured ectoderm cells from Xenopus gastrula. Development 119, 1067-1078.

Kintner, C. R. and Melton, D. A. (1987). Expression of Xenopus NCAM RNA in ectoderm is an early response to neural induction. Development 99 311-325

Knecht, A. K., Good, P. G., Dawid, I. B. and Harland, R. M. (1995). Dorsalventral patterning and differentiation of noggin-induced neural tissue in the asbence of mesoderm. Development 121, 1927-1936.

Knecht, A. K. and Harland, R. M. (1997). Mechanisms of dorsal-ventral patterning in noggin-induced neural tissue. Development 124, 2477-2488

Krieg, P. A., Varnum, S. M., Wormington, W. M. and Melton, D. A. (1989). The mRNA encoding elongation factor 1-alpha (EF-1 alpha) is a major transcript at the midblastula transition in Xenopus. Dev. Biol. 133, 93-100.

LaBonne, C. and Whitman, M. (1994). Mesoderm induction by activin requires FGF-mediated intracellular signals. Development 120, 463-472.

LaBonne, C. and Whitman, M. (1997). Localization of MAP kinase activity in early Xenopus embryos: Implications for endogenous FGF signaling. Developmental Biology 183 9-20.

Lamb, T. M. and Harland, R. M. (1995). Fibroblast growth factor is a direct neural inducer, which combined with noggin generates anterior-posterior neural pattern. Development 121, 3627-3636.

Lamb, T. M., Knecht, A. K., Smith, W. C., Stachel, S. E., Economides, A. N., Stahl, N., Yancopolous, G. D. and Harland, R. M. (1993). Neural induction by the secreted peptide noggin. Science 262, 713-718.

Le Douarin, N. (1982). The Neural Crest. Cambridge: Cambridge University Press.

Liem, K. F., Tremmi, G., Roelink, H. and Jessell, T. M. (1995). Dorsal differentiation of neural plate cells induced by BMP-mediated signals from epidermal ectoderm. Cell 82, 969-979.

Mancilla, A. and Mayor, R. (1996). Neural crest formation in Xenopus laevis: mechanisms of Xslug induction. Dev. Biol. 177, 580-589.

Mayor, R., Morgan, R. and Sargent, M. G. (1995). Induction of the prospective neural crest of Xenopus. Development 121, 767-777.

Mayor, R., Guerrero, N. and Martinez, C. (1997) Role of FGF and noggin in neural crest induction. Dev. Biol. 189, 1-12.

McGrew, L. L., Lai, C.-J. and Moon, R. T. (1995). Specification of the anterioposterior neural axis through synergistic interaction of the Wnt signaling cascade with noggin and follostatin. Dev. Biol., 172, 337-342.

McGrew, L. L., Hoppler, S. and Moon, R. T. (1997) Wnt and FGF pathways cooperatively pattern anteroposterior neural ectoderm in Xenopus. Mech Dev 69, 105-114.

Morgan, R. and Sargent M. G. (1997) The role in neural patterning of translation initiation factor eIF4AII; induction of neural fold genes Development 124 2751-2760. 
Moury, J. D. and Jacobson, A. G. (1990). The origins of neural crest cells in the axolotl. Dev. Biol. 141, 243-253.

Nieto, M. A., Sargent, M. G., Wilkinson, D. G. and Cooke, J. (1994), Control of cell behaviour during vertebrate development by Slug, a zinc finger gene. Science 264, 835-839.

Nieuwkoop, P. D. and Albers, B. (1990). Dev. Growth Differ. 32, 23-31.

Nieuwkoop, P. D. and Faber, J. (1967). Normal Table of Xenopus laevis (Daudin).

Piccolo, S., Sasai, Y., Lu, B. and De Robertis, E. M. (1996). Dorsoventral patterning in Xenopus: inhibition of ventral signals by direct binding of chordin to BMP-4. Cell 86, 589-598.

Poznanski, A., Minsuk, S., Stathopoulos, D. and Keller, R. (1987) Epithelial cell wedging and neural trough formation are induced planarly in Xenopus without persistent vertical interactions with mesoderm. Dev. Biol. 189, 256269.

Raven, C. P. and Kloos, J. (1945). Induction by medial and lateral pieces of the archenteron roof with special reference to the determination of the neural crest. Acta. néerl. Morph. 5, 348-362.

Saint-Jennet, J., He, X., Varmus, H. E. and Dawid, I. B. (1997). Regulation of dorsal fate in the neuraxis by Wnt-1 and Wnt-3a. Proc. Natl. Acad. Sci. USA 94, 13713-13718.

Sasai, Y. and De Robertis, E. M. (1997). Ectodermal patterning in vertebrate embryos. Dev. Biol. 182, 5-20.
Sasai, Y., Lu, B., Steinbesser, H., Geissert, D., Gont, L. K. and De Robertis, E. M. (1994). Xenopus chordin: a novel dorsalizing factor activated by organizer-specific homeobox genes. Cell 79, 779-790.

Savagne, R. P., Yamada, K. M. and Thiery, J. P. (1997) The zinc-finger protein slug causes desmosome dissociation, an initial and necessary step for growth factor-induced epithelial-mesenchymal transition. J. Cell Biol. 137, 1403-1419.

Schroeder, T. E. (1970) Neurulation in Xenopus laevis, an analysis and model based upon light and electron microscopy. J. Embryol. Exp. Morphol. 23, 427-462.

Selleck, M. A. J. and Bronner-Fraser, M. (1995). Origins of the avian neural crest: the role of neural plate-epidermal interactions. Development 121, 525538.

Weinstein, D. C. and Hemmati-Brivanlou, A. (1997). Neural induction in Xenopus laevis: evidence for the default model. Curr. Op. Neurobiol. 7, 7-12.

Wilson, P. A. and Hemmati-Brivanlou, A. (1995). Induction of epidermis and inhibition of neural fate by Bmp-4. Nature 376, 331-333.

Wilson, P. A., Lagna, G., Suzuki, A. and Hemmati-Brivanlou, A. (1997). Concentration-dependent patterning of the Xenopus ectoderm by BMP4 and its signal transducer Smad1. Development 124, 3177-3184.

Zimmerman, L. B., De Jesús-Escobar, J. M. and Harland, R. M. (1996). The Spemann organizer signal noggin binds and inactivates bone morphogenetic protein 4. Cell 86, 599-606. 